\title{
An Iterative Method for the Least-Squares Problems of a General Matrix Equation Subjects to Submatrix Constraints
}

\author{
Li-fang Dai, Mao-lin Liang, and Yong-hong Shen \\ School of Mathematics and Statistics, Tianshui Normal University, Tianshui, Gansu 741001, China \\ Correspondence should be addressed to Li-fang Dai; tsnclml2012@hotmail.com
}

Received 26 July 2013; Accepted 22 October 2013

Academic Editor: Debasish Roy

Copyright (c) 2013 Li-fang Dai et al. This is an open access article distributed under the Creative Commons Attribution License, which permits unrestricted use, distribution, and reproduction in any medium, provided the original work is properly cited.

\begin{abstract}
An iterative algorithm is proposed for solving the least-squares problem of a general matrix equation $\sum_{i=1}^{t} M_{i} Z_{i} N_{i}=F$, where $Z_{i}$ $(i=1,2, \ldots, t)$ are to be determined centro-symmetric matrices with given central principal submatrices. For any initial iterative matrices, we show that the least-squares solution can be derived by this method within finite iteration steps in the absence of roundoff errors. Meanwhile, the unique optimal approximation solution pair for given matrices $\widetilde{Z}_{i}$ can also be obtained by the least-norm least-squares solution of matrix equation $\sum_{i=1}^{t} M_{i} \bar{Z}_{i} N_{i}=\bar{F}$, in which $\bar{Z}_{i}=Z_{i}-\widetilde{Z}_{i}, \bar{F}=F-\sum_{i=1}^{t} M_{i} \widetilde{Z}_{i} N_{i}$. The given numerical examples illustrate the efficiency of this algorithm.
\end{abstract}

\section{Introduction}

Throughout this paper, we denote the set of all $m \times n$ real matrices by $R^{m \times n}$. The symbol $A^{T}$ represents the transpose of matrix $A . J_{n}$ and $I_{n}$ stand for the reverse unit matrix, and identity matrix, respectively. For $A, B \in R^{m \times n}$, the inner product of matrices $A$ and $B$ is defined by $\langle A, B\rangle=$ trace $\left(B^{T} A\right)$, which leads to the Frobenius norm, that is, $\|A\|=$ $\sqrt{\langle A, A\rangle}$.

A matrix $A=\left(a_{i j}\right) \in R^{n \times n}$, is called centro-symmetric (centro-skew symmetric) if and only if

$$
a_{i j}=a_{n-i+1, n-j+1}\left(a_{i j}=-a_{n-i+1, n-j+1}\right), \quad i, j=1,2, \ldots, n,
$$

which can also be characterized equivalently by $J_{n} A J_{n}=$ $A\left(J_{n} A J_{n}=-A\right)$. The set of all centro-symmetric (centroskew symmetric) matrices is denoted by $\operatorname{CS}^{n \times n}\left(\operatorname{CASR}^{n \times n}\right)$. This kind of matrices plays an important role in many applications (see, e.g., [1-4]), and has been frequently and widely investigated (see, e.g., [5-7]) by using generalized inverse, generalized singular value decomposition (GSVD) [8], and so forth. For more, we refer the readers to [9-16] and therein.

We firstly introduce the concept of the central principal submatrix which is originally put forward by Yin [17].
Definition 1. Let $A \in R^{n \times n}$, if $n-p$ is even, a $p \times p$ central principal submatrix of $A$, denoted by $A[p]$, is obtained by deleting the first and last $(n-p) / 2$ rows and columns of $A$, namely, $A[p]=\left(a_{i j}\right)_{(n-p) / 2 \leq i, j \leq n-(n-p) / 2}$.

Evidently, a matrix with odd (even) order only has central principal submatrices of odd (even) order.

Now, the first problem to be studied here can be stated as follows.

Problem 2. Given $M_{i} \in R^{m \times p_{i}}, N_{i} \in R^{p_{i} \times n}$ and $\mathscr{X}_{i} \in \operatorname{CSR}^{q_{i} \times q_{i}}$ $(i=1,2, \ldots, t), F \in R^{m \times n}$. Find the least-squares solution $Z_{i} \in \Pi_{i}$ of matrix equation

$$
M_{1} Z_{1} N_{1}+M_{2} Z_{2} N_{2}+\cdots+M_{t} Z_{t} N_{t}=F,
$$

in which $\Pi_{i}=\left\{Z_{i} \mid Z_{i} \in \mathrm{CSR}^{p_{i} \times p_{i}}\right.$ with $\left.Z_{i}\left[q_{i}\right]=\mathscr{X}_{i}\right\}, p_{i}>q_{i}$, $i \in \Gamma \triangleq\{1,2, \ldots, t\}$, and $Z_{i}\left[q_{i}\right]$ represents the $q_{i} \times q_{i}$ central principal submatrix of $Z_{i}$.

Problem 2 is the submatrix constrained problem of matrix equation (2), which originally arises from a practical subsystem expansion problem, and has been deeply investigated (see, e.g., [7, 18-22]). In these literatures, the generalized inverses or some complicated matrix decompositions such as canonical correlation decomposition (CCD) [23] and GSVD 
are employed. However, it is almost impossible to solve (2) by the above methods. The iterative method is an efficient approach. Recently, kinds of iteration methods have been constructed: Zhou and Duan [24] studied the generalized Sylvester matrix equation

$$
\sum_{i=0}^{\phi} A_{i} X F^{i}+\sum_{k=0}^{\psi} B_{k} Y F^{k}=R
$$

by so-called generalized Sylvester mapping that has pretty properties. Wu et al. [25] presented an finite iterative method for a class of complex matrix equations including conjugate and transpose of unknown solution. Motivated by the wellknown Jacobi and Gauss-Seidel iterations methods, Ding and Chen, in [26], proposed a general family of iterative methods to solve linear matrix equations; meanwhile, these methods were also extended to solve the following coupled Sylvester matrix equations

$$
\sum_{j=1}^{p} A_{i j} X_{j} B_{i j}=C_{i j}, \quad i=1,2, \ldots
$$

Although these iterative algorithms are efficient, there still exist some handicaps when meeting the constrained matrix equation problem (i.e., to find the solution of matrix equation in some matrices sets with specifical structure, for instance, symmetric matrices, centro-symmetric matrices, and bisymmetric matrices sets) and the submatrix constrained problem, since these methods cannot keep the special properties of the unknown matrix in the iterative process. Based on the classical conjugate gradient (CG) method, Peng et al. [27] gave an iterative method to find the bisymmetric solution of matrix equation (2). Similar method was constructed to solve matrix equations (4) with generalized bisymmetric $X_{j}$ in [28]. In particular, Li et al. [29] proposed an elegant algorithm for solving the generalized Sylvester (Lyapunov) matrix equation $A X B+C Y D=E$ with bisymmetric $X$ and symmetric $Y$, the two unknown matrices include the given central principal submatrix and leading principal submatrix, respectively. This method shunned the difficulties in numerical instability and computational complexity, and solved the problem, completely. By borrowing the thinking of this iterative algorithm, we will solve Problem 2 by iteration method.

The second problem to be considered is the optimal approximation problem.

Problem 3. Let $S_{E}$ be the solutions set of Problem 2. For given matrices $\widetilde{Z}_{i} \in R^{p_{i} \times p_{i}}$, find $\widehat{Z}_{i}$ such that

$$
\sum_{i=1}^{t}\left\|\widehat{Z}_{i}-\widetilde{Z}_{i}\right\|^{2}=\min _{\left(Z_{1}, Z_{2}, \ldots, Z_{t}\right) \in S_{E}} \sum_{i=1}^{t}\left\|Z_{i}-\widetilde{Z}_{i}\right\|^{2} .
$$

This problem occurs frequently in experimental design (see for instance [30]). Here, the preliminary estimation $\widetilde{Z}_{i}$ of the unknown matrix $Z_{i}$ can be obtained from experiments, but it may not satisfy the structural requirement and/or spectral requirement. The best estimation of $Z_{i}$, is the matrix
$\widehat{Z}_{i}$ that satisfies both requirements, which is the optimal approximation of $Z_{i}$ (see, e.g., [31, 32]). About this problem, we also refer the authors to [9-11, 13, 15, 16, 20-23, 27-29, 3336] and therein.

The rest of this paper is outlined as follows. In Section 2, an iterative algorithm will be proposed to solve Problem 2, and the properties of which will be investigated. In Section 3, we will consider the optimal approximation Problem 3 by using the iterative algorithm. In Section 4, some numerical examples will be given to verify the efficiency of this algorithm.

\section{The Algorithm for Problem 2 and Its Properties}

According to the definition of centro-symmetric matrix, when $n-q$ is even, a centro-symmetric matrix $Z \in \operatorname{CSR}^{n \times n}$ can be divided into smaller submatrices, namely,

$$
Z=\left(\begin{array}{ccc}
Z_{11} & Z_{12} & Z_{13} \\
Z_{21} & Z_{22} & J_{q} Z_{21} J_{(n-q) / 2} \\
J_{(n-q) / 2} Z_{13} J_{(n-q) / 2} & J_{(n-q) / 2} Z_{12} J_{q} & J_{(n-q) / 2} Z_{11} J_{(n-q) / 2}
\end{array}\right) \text {, }
$$

where $Z_{11} \in R^{(n-q) / 2 \times(n-q) / 2}, Z_{12} \in R^{(n-q) / 2 \times q}, Z_{13} \in$ $R^{(n-q) / 2 \times(n-q) / 2}, Z_{21} \in R^{q \times(n-q) / 2}$, and $Z_{22} \in \mathrm{CSR}^{q \times q}$.

Now, for some fixed positive integer $i \in \Gamma$, we define two matrix sets.

$$
\begin{gathered}
\operatorname{CSR}_{\star, q_{i}}^{p_{i} \times p_{i}}=\left\{Z_{i} \mid Z_{i} \in \mathrm{CSR}^{p_{i} \times p_{i}} \text { with } Z_{i}\left[q_{i}\right]=0\right\}, \\
\operatorname{CS}_{\diamond, q_{i}}^{p_{i} \times p_{i}}=\left\{Z_{i} \mid Z_{i}=\left(\begin{array}{ccc}
0 & 0 & 0 \\
0 & X_{i} & 0 \\
0 & 0 & 0
\end{array}\right) \in \mathrm{CSR}^{p_{i} \times p_{i}},\right. \\
\left.X_{i}=Z_{i}\left[q_{i}\right] \in \mathrm{CSR}^{q_{i} \times q_{i}}\right\} .
\end{gathered}
$$

It is clear that both $\operatorname{CS} R_{\star, q_{i}}^{p_{i} \times p_{i}}$ and $\operatorname{CS} R_{\diamond, q_{i}}^{p_{i} \times p_{i}}$ are linear subspaces of $R^{p_{i} \times p_{i}}$.

In addition, for any matrix $X \in R^{p_{i} \times p_{i}}$, it has uniquely decomposition in direct sum, that is, $X=X_{1} \oplus X_{2}$, here $X_{1}=$ $\left(X+J_{p_{i}} X J_{p_{i}}\right) / 2, X_{2}=\left(X-J_{p_{i}} X J_{p_{i}}\right) / 2$. Furthermore, $X_{1}=$ $X_{11}+X_{12}$ is also the direct sum decomposition of $X_{1}$ if $X_{11} \in$ $\mathrm{CSR}_{\star, q_{i}}^{p_{i} \times p_{i}}, Z_{12} \in \mathrm{CSR}_{\diamond, q_{i}}^{p_{i} \times p_{i}}$, since $\left\langle X_{11}, X_{12}\right\rangle=0$. Hence, we obtain the following.

Lemma 4. Consider $R^{p_{i} \times p_{i}}=C S R_{\star, q_{i}}^{p_{i} \times p_{i}} \oplus C S R_{\diamond, q_{i}}^{p_{i} \times p_{i}} \oplus C A S R^{p_{i} \times p_{i}}$.

Lemma 4 reveals that any matrix $W \in R^{p_{i} \times p_{i}}$ can be uniquely written as $W=W_{1}+W_{2}+W_{3}$, where $W_{1} \in \operatorname{CS} R_{\star, q_{i}}^{p_{i} \times p_{i}}$, $W_{2} \in \operatorname{CSR}_{\diamond, q_{i}}^{p_{i} \times p_{i}}, W_{3} \in \mathrm{CASR}^{p_{i} \times p_{i}}$. Then, we can define the following linear projection operators:

$$
\begin{gathered}
\mathscr{L}_{i}: R^{p_{i} \times p_{i}} \longrightarrow \mathrm{CSR}_{\star, q_{i}}^{p_{i} \times p_{i}} \\
W \longrightarrow W_{1}
\end{gathered}
$$

for $i \in \Gamma$. 
According to the definition of $\mathscr{L}_{i}$, if $W \in R^{p_{i} \times p_{i}}$ and $Y \in$ $\operatorname{CS} R_{\star, q_{i}}^{p_{i} \times p_{i}}$, we have

$$
\langle W, Y\rangle=\left\langle W_{1}, Y\right\rangle=\left\langle\mathscr{L}_{i}(W), Y\right\rangle .
$$

This property will be employed frequently in the residual context.

The following theorem is essential for solving Problem 2, which transforms equivalently Problem 2 into solving the least-square problem of another matrix equation.

Theorem 5. Any solution group of Problem 2 can be obtained by

$$
\left(Z_{1}, Z_{2}, \ldots, Z_{t}\right)=\left(Y_{1}+Z_{1}^{\diamond}, Y_{2}+Z_{2}^{\diamond}, \ldots, Y_{t}+Z_{t}^{\diamond}\right),
$$

where $Y_{i} \in C S R_{\star, q_{i}}^{p_{i} \times p_{i}}$ is the least-squares solution of matrix equation

$$
\begin{gathered}
M_{1} Y_{1} N_{1}+M_{2} Y_{2} N_{2}+\cdots+M_{t} Y_{t} N_{t}=G, \\
G=F-\left(M_{1} Z_{1}^{\diamond} N_{1}+M_{2} Z_{2}^{\diamond} N_{2}+\cdots+M_{t} Z_{t}^{\diamond} N_{t}\right), \\
Z_{i}^{\diamond}=\left(\begin{array}{ccc}
0 & 0 & 0 \\
0 & \mathscr{X}_{i} & 0 \\
0 & 0 & 0
\end{array}\right) \in C S R_{\diamond, q_{i}}^{p_{i} \times p_{i}},
\end{gathered}
$$

$\mathscr{X}_{i} \in \operatorname{CSR}^{q_{i} \times q_{i}}$ is the given central principal submatrix of $Z_{i}$ in Problem 2.

Proof. Noting that the definition of $\operatorname{CS} R_{\star, q_{i}}^{p_{i} \times p_{i}}$, we have

$$
\begin{aligned}
\min _{Z_{i} \in \Pi_{i}}\left\|\sum_{i=1}^{t} M_{i} Z_{i} N_{i}-F\right\| \\
\qquad \min _{Y_{i} \in \operatorname{CS} R_{\star, q_{i}}^{p_{i} \times p_{i}}}\left\|\sum_{i=1}^{t} M_{i}\left(Y_{i}+\bar{Z}_{i}\right) N_{i}-F\right\| \\
\Longleftrightarrow \min _{Y_{i} \in \operatorname{CSR}_{\star q_{i}}^{p_{i} \times q_{i}}}\left\|\sum_{i=1}^{t} M_{i} Y_{i} N_{i}-G\right\| .
\end{aligned}
$$

The proof is completed.

Remark 6. It follows, from Theorem 5, that Problem 2 can be solved completely by finding the least-squares solution of matrix equations (11) in subspaces $\operatorname{CS} R_{\diamond, q_{i}}^{p_{i} \times p_{i}}$.

In the next part of this section, we will establish an iterative algorithm for (11) and analysis its properties. For the convenience of expression, we define a matrix function

$$
\mathscr{F}\left(Z_{1}, Z_{2}, \ldots, Z_{t}\right)=M_{1} Z_{1} N_{1}+M_{2} Z_{2} N_{2}+\cdots+M_{t} Z_{t} N_{t},
$$

then matrix equation (11) can be simplified as

$$
\mathscr{F}\left(Z_{1}, Z_{2}, \ldots, Z_{t}\right)=G .
$$

Moreover, we can easily verify that

$$
\left\langle X, \mathscr{F}\left(Z_{1}, Z_{2}, \ldots, Z_{t}\right)\right\rangle=\sum_{i=1}^{t}\left\langle M_{i}^{T} X N_{i}^{T}, Z_{i}\right\rangle
$$

holds for arbitrary $X \in R^{m \times n}$.
The iterative algorithm for the least squares problem of matrix equations (11) can be expressed as follows.

Algorithm 7. Consider the following.

Step 1. Let $M_{i} \in R^{m \times p_{i}}, N_{i} \in R^{p_{i} \times n}, F \in R^{m \times n}$ and $\mathscr{X}_{i} \in$ $\operatorname{CSR}^{q_{i} \times q_{i}}$ for $i \in \Gamma$.

Input arbitrary matrices $Y_{i}^{(0)} \in \mathrm{CSR}_{\star, q_{i}}^{p_{i} \times p_{i}}$.

Step 2. Calculate

$$
\begin{gathered}
R_{0}=G-\mathscr{F}\left(Y_{1}^{(0)}, Y_{2}^{(0)}, \ldots, Y_{t}^{(0)}\right), \\
P_{i}^{(0)}=\mathscr{L}_{i}\left(M_{i}^{T} R_{0} N_{i}^{T}\right), \quad Q_{i}^{(0)}=P_{i}^{(0)}, \\
k=0 .
\end{gathered}
$$

Step 3. Calculate

$$
\begin{gathered}
Y_{i}^{(k+1)}=Y_{i}^{(k)}+\alpha_{k} Q_{i}^{(k)}, \\
\alpha_{k}=\frac{\sum_{i=1}^{t}\left\|P_{i}^{(k)}\right\|^{2}}{\left\|\mathscr{F}\left(Q_{1}^{(k)}, Q_{2}^{(k)}, \ldots, Q_{t}^{(k)}\right)\right\|^{2}} .
\end{gathered}
$$

Step 4. Calculate

$$
\begin{gathered}
R_{k+1}=G-\mathscr{F}\left(Y_{1}^{(k+1)}, Y_{2}^{(k+1)}, \ldots, Y_{t}^{(k+1)}\right) \\
=R_{k}-\alpha_{k} \mathscr{F}\left(Q_{1}^{(k)}, Q_{2}^{(k)}, \ldots, Q_{t}^{(k)}\right), \\
P_{i}^{(k+1)}=\mathscr{L}_{i}\left(M_{i}^{T} R_{k+1} N_{i}^{T}\right) \\
=P_{i}^{(k)}-\alpha_{k} \mathscr{L}_{i}\left(M_{i}^{T} \mathscr{F}\left(Q_{1}^{(k)}, Q_{2}^{(k)}, \ldots, Q_{t}^{(k)}\right) N_{i}^{T}\right), \\
Q_{i}^{(k+1)}=P_{i}^{(k+1)}+\beta_{k} Q_{i}^{(k)}, \\
\beta_{k}=\frac{\sum_{i=1}^{t}\left\|P_{i}^{(k+1)}\right\|^{2}}{\sum_{i=1}^{t}\left\|P_{i}^{(k)}\right\|^{2}} .
\end{gathered}
$$

Step 5. If $\sum_{i=1}^{t}\left\|P_{i}^{(k)}\right\|^{2}=0$, stop. Otherwise, $k:=k+1$, go to Step 3.

From Algorithm 7, we can see that $Y_{i}^{(k)}, P_{i}^{(k)}, Q_{i}^{(k)}, \epsilon$ $\operatorname{CSR}_{\star, q_{i}}^{p_{i} \times p_{i}}$. In particular, $\left(Y_{1}^{(k)}, Y_{2}^{(k)}, \ldots, Y_{t}^{(k)}\right)$ is a least-squares solution group belonging to matrix equation (11) if $P_{i}^{(k)}=0$ for all $i \in \Gamma$. The following lemma gives voice to the reason.

Lemma 8. If $\mathscr{L}_{i}\left(M_{i}^{T} R_{k} N_{i}^{T}\right)=0(i=1,2, \ldots, t)$ hold simultaneously for some positive $k$, then $\left(Y_{1}^{(k)}, Y_{2}^{(k)}, \ldots, Y_{t}^{(k)}\right)$ generated by Algorithm 7 is a solution group of matrix equation (11).

Proof. Let $\mathscr{L}=\left\{L \mid L=\mathscr{F}\left(Y_{i}\right), Y_{i} \in \operatorname{CSR}_{\star, q_{i}}^{p_{i} \times p_{i}}\right\}$ and $\widehat{G}=$ $\mathscr{F}\left(Y_{i}^{(k)}\right)$. Obviously, $\widehat{G} \in \mathscr{L}$. Then, from the Project Theorem, $\left(Y_{1}^{(k)}, Y_{2}^{(k)}, \ldots, Y_{t}^{(k)}\right)$ is a least-square solution group of matrix equation (11) if and only if $G-\widehat{G} \perp \mathscr{L}$. That is to say, for 


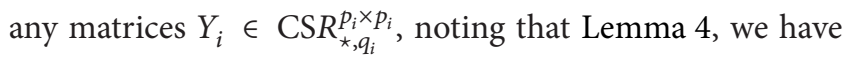
$\left\langle G-\widehat{G}, \mathscr{F}\left(Y_{1}, Y_{2}, \ldots, Y_{t}\right)\right\rangle=0$, that is,

$$
\begin{aligned}
\left\langle G-\widehat{G}, \mathscr{F}\left(Y_{1}, Y_{2}, \ldots, Y_{t}\right)\right\rangle & =\left\langle R_{k+1}, \mathscr{F}\left(Y_{1}, Y_{2}, \ldots, Y_{t}\right)\right\rangle \\
& =\sum_{i=1}^{t}\left\langle M_{i}^{T} R_{k+1} N_{i}^{T}, Y_{i}\right\rangle \\
& =\sum_{i=1}^{t}\left\langle\mathscr{L}_{i}\left(M_{i}^{T} R_{k+1} N_{i}^{T}\right), Y_{i}\right\rangle=0,
\end{aligned}
$$

which completes the proof.

In addition, the sequences $\left\{Y_{i}^{(l)}\right\},\left\{P_{i}^{(l)}\right\},\left\{Q_{i}^{(l)}\right\}$ generated by Algorithm 7 are self-orthogonal, that is, as follows.

Lemma 9. Suppose that the sequences $\left\{Y_{i}^{(l)}\right\},\left\{P_{i}^{(l)}\right\},\left\{Q_{i}^{(l)}\right\}$ generated by Algorithm 7 not equal null for $l \leq k$, then
(1) $\sum_{i=1}^{t}\left\langle P_{i}^{(j)}, P_{i}^{(l)}\right\rangle=0$
(2) $\sum_{i=1}^{t}\left\langle Q_{i}^{(j)}, P_{i}^{(l)}\right\rangle=0$,
(3) $\left\langle\mathscr{F}\left(Q_{1}^{(j)}, Q_{2}^{(j)}, \ldots, Q_{t}^{(j)}\right), \mathscr{F}\left(Q_{1}^{(l)}, Q_{2}^{(l)}, \ldots, Q_{t}^{(l)}\right)\right\rangle=0$,

where $j, l=1,2, \ldots, k, j \neq l \leq k$.

Proof. In view of the symmetry of the inner product, we only prove (20)-(22) when $j<l$. According to Algorithm 7, when $k=1$, we have

$$
\begin{aligned}
& \sum_{i=1}^{t}\left\langle P_{i}^{(0)}, P_{i}^{(1)}\right\rangle \\
& =\sum_{i=1}^{t}\left\langle P_{i}^{(0)}, P_{i}^{(0)}-\alpha_{0} \mathscr{L}_{i} M_{i}^{T}\left(\mathscr{F}\left(Q_{1}^{(0)}, Q_{2}^{(0)}, \ldots, Q_{t}^{(0)}\right) N_{i}^{T}\right)\right\rangle \\
& =\sum_{i=1}^{t}\left[\left\langle P_{i}^{(0)}, P_{i}^{(0)}\right\rangle\right. \\
& \left.\quad-\alpha_{0}\left\langle P_{i}^{(0)}, \mathscr{L}_{i} M_{i}^{T}\left(\mathscr{F}\left(Q_{1}^{(0)}, Q_{2}^{(0)}, \ldots, Q_{t}^{(0)}\right) N_{i}^{T}\right)\right\rangle\right] \\
& =\sum_{i=1}^{t}\left\|P_{i}^{(0)}\right\|^{2}-\alpha_{0} \\
& \quad \times \sum_{i=1}^{t}\left\langle P_{i}^{(0)}, M_{i}^{T} \mathscr{F}\left(Q_{1}^{(0)}, Q_{2}^{(0)}, \ldots, Q_{t}^{(0)}\right) N_{i}^{T}\right\rangle
\end{aligned}
$$

$$
\begin{aligned}
& =\sum_{i=1}^{t}\left\|P_{i}^{(0)}\right\|^{2}-\alpha_{0} \sum_{i=1}^{t}\left\langle M_{i} P_{i}^{(0)} N_{i}, \mathscr{F}\left(Q_{1}^{(0)}, Q_{2}^{(0)}, \ldots, Q_{t}^{(0)}\right)\right\rangle \\
& =\sum_{i=1}^{t}\left\|P_{i}^{(0)}\right\|^{2}-\alpha_{0}\left\|\mathscr{F}\left(Q_{1}^{(0)}, Q_{2}^{(0)}, \ldots, Q_{t}^{(0)}\right)\right\|^{2} \\
& =0
\end{aligned}
$$

which also deduces that

$$
\sum_{i=1}^{t}\left\langle Q_{i}^{(0)}, P_{i}^{(1)}\right\rangle
$$

$$
\begin{aligned}
= & \sum_{i=1}^{t}\left\langle Q_{i}^{(0)}, P_{i}^{(0)}-\alpha_{0} \mathscr{L}_{i}\left(M_{i}^{T} \mathscr{F}\left(Q_{1}^{(0)}, Q_{2}^{(0)}, \ldots, Q_{t}^{(0)}\right) N_{i}^{T}\right)\right\rangle \\
= & \sum_{i=1}^{t}\left\langle Q_{i}^{(0)}, P_{i}^{(0)}\right\rangle-\alpha_{0} \\
& \times \sum_{i=1}^{t}\left\langle Q_{i}^{(0)}, M_{i}^{T} \mathscr{F}\left(Q_{1}^{(0)}, Q_{2}^{(0)}, \ldots, Q_{t}^{(0)}\right) N_{i}^{T}\right\rangle \\
= & \sum_{i=1}^{t}\left\langle P_{i}^{(0)}, P_{i}^{(0)}\right\rangle-\alpha_{0} \\
& \times \sum_{i=1}^{t}\left\langle M_{i} Q_{i}^{(0)} N_{i}, \mathscr{F}\left(Q_{1}^{(0)}, Q_{2}^{(0)}, \ldots, Q_{t}^{(0)}\right)\right\rangle
\end{aligned}
$$

$=0$,

$$
\left\langle\mathscr{F}\left(Q_{1}^{(0)}, Q_{2}^{(0)}, \ldots, Q_{t}^{(0)}\right), \mathscr{F}\left(Q_{1}^{(1)}, Q_{2}^{(1)}, \ldots, Q_{t}^{(1)}\right)\right\rangle
$$

$$
=\left\langle\mathscr{F}\left(Q_{1}^{(0)}, Q_{2}^{(0)}, \ldots, Q_{t}^{(0)}\right), \sum_{i=1}^{t}\left[M_{i}\left(P_{i}^{(1)}+\beta_{0} Q_{i}^{(0)}\right) N_{i}\right]\right\rangle
$$$$
=\left\langle\mathscr{F}\left(Q_{1}^{(0)}, Q_{2}^{(0)}, \ldots, Q_{t}^{(0)}\right), \mathscr{F}\left(P_{1}^{(1)}, P_{2}^{(1)}, \ldots, P_{t}^{(1)}\right)\right\rangle
$$$$
+\beta_{0}\left\|\mathscr{F}\left(Q_{1}^{(0)}, \ldots, Q_{t}^{(0)}\right)\right\|^{2}
$$$$
=\frac{1}{\alpha_{0}}\left\langle R_{0}-R_{1}, \mathscr{F}\left(P_{1}^{(1)}, P_{2}^{(1)}, \ldots, P_{t}^{(1)}\right)\right\rangle
$$$$
+\beta_{0}\left\|\mathscr{F}\left(Q_{1}^{(0)}, Q_{2}^{(0)}, \ldots, Q_{t}^{(0)}\right)\right\|^{2}
$$$$
=\frac{1}{\alpha_{0}}\left[\sum_{i=1}^{t}\left\langle M_{i}^{T} R_{0} N_{i}^{T}, P_{i}^{(1)}\right\rangle-\sum_{i=1}^{t}\left\langle M_{i}^{T} R_{1} N_{i}^{T}, P_{i}^{(1)}\right\rangle\right]
$$$$
+\beta_{0}\left\|\mathscr{F}\left(Q_{1}^{(0)}, Q_{2}^{(0)}, \ldots, Q_{t}^{(0)}\right)\right\|^{2}
$$$$
=-\frac{1}{\alpha_{0}} \sum_{i=1}^{t}\left\|P_{i}^{(1)}\right\|^{2}+\beta_{0}\left\|\mathscr{F}\left(Q_{1}^{(0)}, Q_{2}^{(0)}, \ldots, Q_{t}^{(0)}\right)\right\|^{2}
$$

$=0$. 
Assume that (20), (21), and (22) hold for positive integer $s(<k)$, that is, for $j=1,2, \ldots, s-1$,

$$
\begin{gathered}
\sum_{i=1}^{t}\left\langle P_{i}^{(j)}, P_{i}^{(s)}\right\rangle=0, \\
\sum_{i=1}^{t}\left\langle Q_{i}^{(j)}, P_{i}^{(s)}\right\rangle=0, \\
\left\langle\mathscr{F}\left(Q_{1}^{(j)}, Q_{2}^{(j)}, \ldots, Q_{t}^{(j)}\right), \mathscr{F}\left(Q_{1}^{(s)}, Q_{2}^{(s)}, \ldots, Q_{t}^{(s)}\right)\right\rangle=0 .
\end{gathered}
$$

Then, similar to the above proof, noting that the assumptions, we have

$$
\begin{aligned}
\sum_{i=1}^{t}\left\langle P_{i}^{(j)}, P_{i}^{(s+1)}\right\rangle & \\
= & \sum_{i=1}^{t}\left\langle P_{i}^{(j)}, P_{i}^{(s)}-\alpha_{s} \mathscr{L}_{i}\left(M_{i}^{T} \mathscr{F}\left(Q_{1}^{(s)}, Q_{2}^{(s)}, \ldots, Q_{t}^{(s)}\right) N_{i}^{T}\right)\right\rangle \\
= & \sum_{i=1}^{t}\left\langle P_{i}^{(j)}, P_{i}^{(s)}\right\rangle-\alpha_{s} \\
& \times \sum_{i=1}^{t}\left\langle P_{i}^{(j)}, \mathscr{L}_{i}\left(M_{i}^{T} \mathscr{F}\left(Q_{1}^{(s)}, Q_{2}^{(s)}, \ldots, Q_{t}^{(s)}\right) N_{i}^{T}\right)\right\rangle \\
= & -\alpha_{s} \sum_{i=1}^{t}\left\langle M_{i} P_{i}^{(j)} N_{i}, \mathscr{F}\left(Q_{1}^{(s)}, Q_{2}^{(s)}, \ldots, Q_{t}^{(s)}\right)\right\rangle \\
= & -\alpha_{s} \sum_{i=1}^{t}\left\langle M_{i}\left(Q_{i}^{(j)}-\beta_{j-1} Q_{j-1, i}\right) N_{i},\right. \\
& +\alpha_{s} \beta_{j-1}\left\langle\mathscr{F}\left(Q_{1}^{(j-1)}, Q_{2}^{(j-1)}, \ldots, Q_{t}^{(s)}, \ldots, Q_{t}^{(s)}\right)\right\rangle \\
= & \left.\mathscr{F}\left(Q_{1}^{(s)}, Q_{2}^{(s)}, \ldots, Q_{t}^{(s)}\right)\right\rangle \\
= & \left\langle\mathscr{F}\left(Q_{1}^{(j)}, Q_{2}^{(j)}, \ldots, Q_{t}^{(j)}\right), \mathscr{F}\left(Q_{1}^{(s)}, Q_{2}^{(s)}, \ldots, Q_{t}^{(s)}\right)\right\rangle \\
& \\
& \\
&
\end{aligned}
$$

Furthermore,

$$
\begin{aligned}
& \sum_{i=1}^{t}\left\langle Q_{i}^{(j)}, P_{i}^{(s+1)}\right\rangle \\
& =\sum_{i=1}^{t}\left\langle Q_{i}^{(j)}, P_{i}^{(s)}-\alpha_{s} \mathscr{L}_{i}\left(M_{i}^{T} \mathscr{F}\left(Q_{1}^{(s)}, Q_{2}^{(s)}, \ldots, Q_{t}^{(s)}\right) N_{i}^{T}\right)\right\rangle \\
& =\sum_{i=1}^{t}\left\langle P_{i}^{(j)}, P_{i}^{(s)}\right\rangle-\alpha_{s}
\end{aligned}
$$

$$
\begin{aligned}
& \times \sum_{i=1}^{t}\left\langle M_{i} Q_{i}^{(j)} N_{i}, \mathscr{F}\left(Q_{1}^{(s)}, Q_{2}^{(s)}, \ldots, Q_{t}^{(s)}\right)\right\rangle \\
= & 0, \\
\langle\mathscr{F} & \left.\left(Q_{1}^{(j)}, Q_{2}^{(j)}, \ldots, Q_{t}^{(j)}\right), \mathscr{F}\left(Q_{1}^{(s+1)}, Q_{2}^{(s+1)}, \ldots, Q_{t}^{(s+1)}\right)\right\rangle \\
= & \left\langle\mathscr{F}\left(Q_{1}^{(j)}, Q_{2}^{(j)}, \ldots, Q_{t}^{(j)}\right), \mathscr{F}\left(P_{1}^{(s+1)}, P_{2}^{(s+1)}, \ldots, P_{t}^{(s+1)}\right)\right\rangle \\
& +\beta_{s}\left\langle\mathscr{F}\left(Q_{1}^{(j)}, Q_{2}^{(j)}, \ldots, Q_{t}^{(j)}\right), \mathscr{F}\left(Q_{1}^{(s)}, Q_{2}^{(s)}, \ldots, Q_{t}^{(s)}\right)\right\rangle \\
= & \frac{1}{\alpha_{j}}\left\langle R_{j}-R_{j+1}, \mathscr{F}\left(P_{1}^{(s+1)}, P_{2}^{(s+1)}, \ldots, P_{t}^{(s+1)}\right)\right\rangle \\
= & \frac{1}{\alpha_{j}}\left[\sum_{i=1}^{t}\left\langle M_{i}^{T} R_{j} N_{i}^{T}, P_{i}^{(s+1)}\right\rangle-\sum_{i=1}^{t}\left\langle M_{i}^{T} R_{j+1} N_{i}^{T}, P_{i}^{(s+1)}\right\rangle\right] \\
= & \frac{1}{\alpha_{j}}\left[\sum_{i=1}^{t}\left\langle P_{i}^{(j)}, P_{i}^{(s+1)}\right\rangle-\sum_{i=1}^{t}\left\langle P_{j+1, i}, P_{i}^{(s+1)}\right\rangle\right] \\
= & 0 .
\end{aligned}
$$

The last equal sign "=" holds due to $\sum_{i=1}^{t}\left\langle P_{i}^{(s)}, P_{i}^{(s+1)}\right\rangle=0$. In fact, from the hypothesis, we deduce

$$
\begin{aligned}
\sum_{i=1}^{t}\left\langle P_{i}^{(s)}, P_{i}^{(s+1)}\right\rangle & \\
= & \sum_{i=1}^{t}\left\langle P_{i}^{(s)}, P_{i}^{(s)}-\alpha_{s} \mathscr{L}_{i}\left(M_{i}^{T} \mathscr{F}\left(Q_{1}^{(s)}, Q_{2}^{(s)}, \ldots, Q_{t}^{(s)}\right) N_{i}^{T}\right)\right\rangle \\
= & \sum_{i=1}^{t}\left\|P_{i}^{(s)}\right\|^{2} \\
& -\alpha_{s} \sum_{i=1}^{t}\left\langle M_{i}\left(Q_{i}^{(s)}-\beta_{s-1} Q_{i}^{(j-1)}\right) N_{i}, \mathscr{F}\left(Q_{1}^{(s)}, Q_{2}^{(s)}, \ldots, Q_{t}^{(s)}\right)\right\rangle \\
= & \sum_{i=1}^{t}\left\|P_{i}^{(s)}\right\|^{2}-\alpha_{s}\left\|\mathscr{F}\left(Q_{1}^{(s)}, Q_{2}^{(s)}, \ldots, Q_{t}^{(s)}\right)\right\|^{2} \\
& +\alpha_{s} \beta_{s-1}\left\langle\mathscr{F}\left(Q_{1}^{(s-1)}, Q_{2}^{(s-1)}, \ldots, Q_{t}^{(s-1)}\right),\right. \\
\left.\mathscr{F}\left(Q_{1}^{(s)}, Q_{2}^{(s)}, \ldots, Q_{t}^{(s)}\right)\right\rangle & 0 .
\end{aligned}
$$

Moreover,

$$
\begin{aligned}
& \sum_{i=1}^{t}\left\langle Q_{i}^{(s)}, P_{i}^{(s)}\right\rangle \\
& \quad=\sum_{i=1}^{t}\left\langle P_{i}^{(s)}+\beta_{s-1} Q_{i}^{(s-1)}, P_{i}^{(s)}\right\rangle
\end{aligned}
$$




$$
\begin{aligned}
& =\sum_{i=1}^{t}\left\|P_{i}^{(s)}\right\|^{2}+\beta_{s-1} \sum_{i=1}^{t}\left\langle Q_{i}^{(s-1)}, P_{i}^{(s)}\right\rangle \\
& =\sum_{i=1}^{t}\left\|P_{i}^{(s)}\right\|^{2} .
\end{aligned}
$$

It follows from (29) that

$$
\begin{aligned}
& \sum_{i=1}^{t}\left\langle Q_{i}^{(s)}, P_{i}^{(s+1)}\right\rangle \\
&= \sum_{i=1}^{t}\left\langle Q_{i}^{(s)}, P_{i}^{(s)}-\alpha_{s} \mathscr{L}_{i}\left(M_{i}^{T} \mathscr{F}\left(Q_{1}^{(s)}, Q_{2}^{(s)}, \ldots, Q_{t}^{(s)}\right) N_{i}^{T}\right\rangle\right. \\
&= \sum_{i=1}^{t}\left[\left\langle Q_{i}^{(s)}, P_{i}^{(s)}\right\rangle\right. \\
&\left.\quad-\alpha_{s}\left\langle Q_{i}^{(s)}, M_{i}^{T} \mathscr{F}\left(Q_{1}^{(s)}, Q_{2}^{(s)}, \ldots, Q_{t}^{(s)}\right) N_{i}^{T}\right\rangle\right] \\
&=\sum_{i=1}^{t}\left\|P_{i}^{(s)}\right\|^{2}-\alpha_{s}\left\|\mathscr{F}\left(Q_{1}^{(s+1)}, Q_{2}^{(s+1)}, \ldots, Q_{t}^{(s+1)}\right)\right\|^{2} \\
&=0, \\
&\left\langle\mathscr{F}\left(Q_{1}^{(s)}, Q_{2}^{(s)}, \ldots, Q_{t}^{(s)}\right), \mathscr{F}\left(Q_{1}^{(s+1)}, Q_{2}^{(s+1)}, \ldots, Q_{t}^{(s+1)}\right)\right\rangle \\
&=\left\langle\mathscr{F}\left(Q_{1}^{(s)}, \ldots, Q_{t}^{(s)}\right), \mathscr{F}\left(P_{1}^{(s+1)}, P_{2}^{(s+1)}, \ldots, P_{t}^{(s+1)}\right)\right\rangle \\
&+\beta_{s}\left\|\mathscr{F}\left(Q_{1}^{(s)}, \ldots, Q_{t}^{(s)}\right)\right\|^{2} \\
&= \frac{1}{\alpha_{s}}\left\langle R_{s}-R_{s+1}, \mathscr{F}\left(P_{1}^{(s+1)}, P_{2}^{(s+1)}, \ldots, P_{t}^{(s+1)}\right)\right\rangle \\
&+\beta_{s} \sum_{i=1}^{t}\left\|M_{i} Q_{i}^{(s)} N_{i}\right\|^{2} \\
&=-\frac{1}{\alpha_{s}} \sum_{i=1}^{t}\left\langle M_{i}^{T} R_{s+1} N_{i}^{T}, P_{i}^{(s+1)}\right\rangle \\
&+\beta_{s} \sum_{i=1}^{t}\left\|M_{i} Q_{i}^{(s)} N_{i}\right\|^{2} \\
&= 0 .
\end{aligned}
$$

Therefore, for arbitrary integers number $l$, the conclusions hold. The proof is completed.
Remark 10. We know from Lemma 4 that the matrices sequences

$$
\begin{gathered}
\left(\begin{array}{ccc}
P_{0,1} & & \\
& \ddots & \\
& & P_{0, t}
\end{array}\right),\left(\begin{array}{lll}
P_{1,1} & & \\
& \ddots & \\
& & P_{1, t}
\end{array}\right), \ldots, \\
\\
\left(\begin{array}{ccc}
P_{k, 1} & & \\
& \ldots & \\
& & P_{k, t}
\end{array}\right), \ldots
\end{gathered}
$$

are orthogonal to each other. Hence, it can be regarded as an orthogonal basis of matrix space $R^{\sum_{i=1}^{t} p_{i} \times \sum_{i=1}^{t} p_{i}}$. Hence, the iteration will be terminated at most $\sum_{i=1}^{t}\left(p_{i}+q_{i}\right)\left(p_{i}-q_{i}\right) / 2$ steps in the absence of roundoff errors. Therefore, there exists a positive integer $k \leq \sum_{i=1}^{t}\left(p_{i}+q_{i}\right)\left(p_{i}-q_{i}\right) / 2$ such that $\sum_{i=1}^{t}\left\|P_{i}^{(k)}\right\|^{2}=0$, in this case, $\left(Y_{1}^{(k)}, Y_{2}^{(k)}, \ldots, Y_{t}^{(k)}\right)$ can be regarded as a least-squares solution group of matrix equation (11).

In addition, we should point out that if $\alpha_{k}=0$ or $\infty$, the conclusions may not be true, and the iteration will break down before $P_{i}^{(k)}=0$ for $k<\sum_{i=1}^{t}\left(p_{i}+q_{i}\right)\left(p_{i}-q_{i}\right) / 2$. Actually, $\alpha_{k}=0$ implies that $\sum_{i=1}^{t}\left\|P_{i}^{(k)}\right\|^{2}=0$, so $P_{i}^{(k)}=0$ for $i \in \Gamma$. While $\alpha_{k}=\infty$ leads to $\mathscr{F}\left(Q_{1}^{(k)}, Q_{2}^{(k)}, \ldots, Q_{t}^{(k)}\right)=0$, making inner product with $R_{i}$ by both sides, it follows from Algorithm 7 that

$$
\begin{aligned}
& \left\langle R_{i}, \mathscr{F}\left(Q_{1}^{(k)}, Q_{2}^{(k)}, \ldots, Q_{t}^{(k)}\right)\right\rangle \\
& =\sum_{i=1}^{t}\left\langle P_{i}^{(k)}, P_{i}^{(k)}+\beta_{k-1} Q_{i}^{(k-1)}\right\rangle \\
& =\sum_{i=1}^{t}\left\|P_{i}^{(k)}\right\|^{2}+\beta_{k-1} \sum_{i=1}^{t}\left\langle P_{i}^{(k)}, Q_{i}^{(k-1)}\right\rangle \\
& =\sum_{i=1}^{t}\left\|P_{i}^{(k)}\right\|^{2}=0,
\end{aligned}
$$

which also implies the same situation as $\alpha_{k}=0$. Hence, if there exists a positive integer $s$ such that the coefficient $\alpha_{s}=0$ or $\alpha_{s}=\infty$, then the corresponding matrix group $Y_{1}^{(s)}, Y_{2}^{(s)}, \ldots, Y_{t}^{(s)}$ is just the solution of matrix equation (11).

Together with the above analysis and Lemma 9, we can conclude the following theorem.

Theorem 11. For any initial iteration matrices $Y_{i}^{(0)} \in C S R_{\star, q_{i}}^{n \times n}$, $i=1,2, \ldots, t$, the least-squares solution of matrix equation (11) can be obtained within finite iteration steps. Moreover, Suppose that $\left(\breve{Y}_{1}, \breve{Y}_{2}, \ldots, \breve{Y}_{t}\right)$ is a least-squares solution group of (11), then the general solution to Problem 2 can be expressed as $Z_{i}=\breve{Y}_{i}+X_{i}+Z_{i}^{\diamond}(i=1,2, \ldots, t)$, where $X_{i} \in C S R_{\star, q_{i}}^{n \times n}$ satisfy homogeneous equation

$$
\mathscr{F}\left(X_{1}, X_{2}, \ldots, X_{t}\right)=0,
$$

$Z_{i}^{\diamond}$ as in Theorem 5 . 
In order to show the validity of Theorem 11, it is adequate to prove the following conclusion.

Proposition 12. The least-squares solution group $\left(\breve{Y}_{1}, \breve{Y}_{2}, \ldots, \breve{Y}_{t}\right)$ of matrix equation (11) can be expressed as $\breve{Y}_{i}+X_{i}$, where $X_{i}$ satisfy equality (33).

Proof. According to the assumptions, we obtain

$$
\begin{array}{r}
\min _{Y_{i} \in \operatorname{CSR}_{\star, q_{i}}^{n \times n}}\left\|\mathscr{F}\left(Y_{1}, Y_{2}, \ldots, Y_{t}\right)-G\right\| \\
=\left\|\mathscr{F}\left(\breve{Y}_{1}, \breve{Y}_{2}, \ldots, \breve{Y}_{t}\right)-G\right\| .
\end{array}
$$

On the other hand, noting that $\mathscr{F}\left(X_{1}, X_{2}, \ldots, X_{t}\right)=0$, then

$$
\begin{aligned}
\| \mathscr{F} & \left(\breve{Y}_{1}+X_{1}, \breve{Y}_{2}+X_{2}, \ldots, \breve{Y}_{t}+X_{t}\right)-G \|^{2} \\
& =\left\|\mathscr{F}\left(\breve{Y}_{1}, \breve{Y}_{2}, \ldots, \breve{Y}_{t}\right)-G+\mathscr{F}\left(X_{1}, X_{2}, \ldots, X_{t}\right)\right\|^{2} \\
& =\left\|\mathscr{F}\left(\breve{Y}_{1}, \breve{Y}_{2}, \ldots, \breve{Y}_{t}\right)-G\right\|^{2} .
\end{aligned}
$$

The proof is completed.

Next, we will show that the unique least norm solution of matrix equation (11) can be derived by choosing a special kind of initial iteration matrices.

Theorem 13. Let the initial iteration matrices $Y_{i}^{(0)}=$ $\mathscr{L}_{i}\left(M_{i}^{T} H N_{i}^{T}\right)$ with arbitrary $H \in R^{m \times n}, i=1,2, \ldots, t$, and then $\left(Y_{1}^{*}, Y_{2}^{*}, \ldots, Y_{t}^{*}\right)$ generated by Algorithm 7 is the leastnorm least-squares solution group of matrix equation (11). Furthermore, the least-norm solution group to Problem 2 can be expressed by

$$
\left(Z_{1}^{*}, Z_{2}^{*}, \ldots, Z_{t}^{*}\right)=\left(Y_{1}^{*}+Z_{i}^{\diamond}, Y_{2}^{*}+Z_{2}^{\diamond}, \ldots, Y_{t}^{*}+Z_{t}^{\diamond}\right) .
$$

Proof. From Algorithm 7 and Theorem 11, for initial iteration matrices $Y_{i}^{(0)}=\mathscr{L}_{i}\left(M_{i}^{T} H N_{i}^{T}\right)$, we can obtain a least-squares solution $Y_{i}^{*}$ of matrix equation (11) and there exists a matrix $H^{*}$ such that $Y_{i}^{*}=\mathscr{L}_{i}\left(M_{i}^{T} H^{*} N_{i}^{T}\right)$. Hence, it is enough to prove that the $Y_{i}^{*}$ is the least-norm solution. In fact, noting that (33) and Proposition 12, we have

$$
\begin{aligned}
& \sum_{i=1}^{t}\left\|Y_{i}^{*}+X_{i}\right\|^{2} \\
& \quad=\sum_{i=1}^{t}\left(\left\|Y_{i}^{*}\right\|^{2}+\left\|X_{i}\right\|^{2}+2\left\langle Y_{i}^{*}, X_{i}\right\rangle\right) \\
& \quad=\sum_{i=1}^{t}\left\|Y_{i}^{*}\right\|^{2}+\sum_{i=1}^{t}\left\|X_{i}\right\|^{2}+2 \sum_{i=1}^{t}\left\langle\mathscr{L}_{i}\left(M_{i}^{T} H^{*} N_{i}^{T}\right), X_{i}\right\rangle \\
& \quad \geq \sum_{i=1}^{t}\left\|Y_{i}^{*}\right\|^{2},
\end{aligned}
$$

as required.
Theorems 11 and 13 display the efficiency of Algorithm 7. Actually, the iteration sequence $\left\{Y_{i}^{(k)}\right\}$ converges smoothly to the solution $Y_{i}$, that is the minimization property of Algorithm 7.

Theorem 14. For any initial iteration matrices $Y_{i}^{(0)}$, the $Y_{i}^{(k)}$ generated by Algorithm 7 satisfy the minimization problem

$$
\begin{aligned}
\left\|\mathscr{F}\left(Y_{1}^{(k)}, Y_{2}^{(k)}, \ldots, Y_{t}^{(k)}\right)-G\right\| \\
\quad=\min _{Y_{i} \in \mathbb{L}_{i}}\left\|\mathscr{F}\left(Y_{1}, Y_{2}, \ldots, Y_{t}\right)-G\right\|,
\end{aligned}
$$

where $\mathbb{L}_{i}=Y_{i}^{(0)}+\operatorname{span}\left\{Q_{i}^{(0)}, Q_{i}^{(1)}, \ldots, Q_{i}^{(k-1)}\right\}, i=1,2, \ldots, t$.

Proof. From the definition of $\mathbb{L}_{i}$, there exist a series of real numbers $a_{0}, a_{1}, \ldots, a_{k-1}$ such that $Y_{i}=Y_{i}^{(0)}+\sum_{l=0}^{k-1} a_{l} Q_{i}^{(l)}$.

Define a function of $k$ variables $f\left(a_{0}, a_{1}, \ldots, a_{k-1}\right)$, that is,

$$
\begin{aligned}
f & \left(a_{0}, a_{1}, \ldots, a_{k-1}\right) \\
& =\left\|\sum_{i=1}^{t}\left[M_{i}\left(Y_{i}^{(0)}+\sum_{l=0}^{k-1} a_{l} Q_{i}^{(l)}\right) N_{i}-G\right]\right\|^{2} .
\end{aligned}
$$

In addition, from Algorithm 7, we know that

$$
\begin{aligned}
R_{0}= & R_{l}+\alpha_{l-1} \mathscr{F}\left(Q_{1}^{(l-1)}, Q_{2}^{(l-1)}, \ldots, Q_{t}^{(l-1)}\right) \\
& +\cdots+\alpha_{0} \mathscr{F}\left(Q_{1}^{(0)}, Q_{2}^{(0)}, \ldots, Q_{t}^{(0)}\right) .
\end{aligned}
$$

Noting that (22) and making the inner product with $\mathscr{F}\left(Q_{1}^{(l)}, Q_{2}^{(l)}, \ldots, Q_{t}^{(l)}\right)$ on both sides of $(40)$ yield

$$
\left\langle\mathscr{F}\left(Q_{1}^{(l)}, Q_{2}^{(l)}, \ldots, Q_{t}^{(l)}\right), R_{0}\right\rangle=\left\langle\mathscr{F}\left(Q_{1}^{(l)}, Q_{2}^{(l)}, \ldots, Q_{t}^{(l)}\right), R_{l}\right\rangle
$$

Hence, by simple calculation, (40) and (41), the function can be rewritten as

$$
\begin{aligned}
f( & \left(a_{0}, a_{1}, \ldots, a_{k-1}\right) \\
= & \left\|R_{0}\right\|^{2}+\sum_{l=0}^{k-1} a_{l}^{2}\left\|\mathscr{F}\left(Q_{1}^{(l)}, Q_{2}^{(l)}, \ldots, Q_{t}^{(l)}\right)\right\|^{2} \\
& -2 \sum_{l=0}^{k-1} a_{l}\left\langle\mathscr{F}\left(Q_{1}^{(l)}, Q_{2}^{(l)}, \ldots, Q_{t}^{(l)}\right), R_{l}\right\rangle .
\end{aligned}
$$

Then,

$$
\begin{aligned}
\min _{a_{l}} f\left(a_{0}, a_{1}, \ldots, a_{k-1}\right) & \\
& \Longleftrightarrow \min _{Y_{i} \in \mathbb{L}_{i}}\left\|\mathscr{F}\left(Y_{1}, Y_{2}, \ldots, Y_{t}\right)-G\right\| .
\end{aligned}
$$


Since $f\left(a_{0}, a_{1}, \ldots, a_{k-1}\right)=\min$ only if $\partial f / \partial a_{l}=0$, it follows from (29) that

$$
\begin{aligned}
a_{l} & =\frac{\left\langle\mathscr{F}\left(Q_{1}^{(l)}, Q_{2}^{(l)}, \ldots, Q_{t}^{(l)}\right), R_{l}\right\rangle}{\left\|\mathscr{F}\left(Q_{1}^{(l)}, Q_{2}^{(l)}, \ldots, Q_{t}^{(l)}\right)\right\|^{2}} \\
& =\frac{\sum_{i=1}^{t}\left\langle Q_{i}^{(l)}, P_{l, i}\right\rangle}{\left\|\mathscr{F}\left(Q_{1}^{(l)}, Q_{2}^{(l)}, \ldots, Q_{t}^{(l)}\right)\right\|^{2}} \\
& =\frac{\sum_{i=1}^{t}\left\|P_{l, i}\right\|^{2}}{\left\|\mathscr{F}\left(Q_{1}^{(l)}, Q_{2}^{(l)}, \ldots, Q_{t}^{(l)}\right)\right\|^{2}}=\alpha_{l} .
\end{aligned}
$$

Combined with (43), we complete the proof.

Theorem 14 reveals that the sequence

$$
\left\{\left\|\mathscr{F}\left(Y_{1}^{(k)}, Y_{2}^{(k)}, \ldots, Y_{t}^{(k)}\right)-G\right\|\right\}
$$

monotonically decreases with respect to increasing integer $k$. The descent property of the residual norm of matrix equation (11) leads to the smoothly convergence of Algorithm 7.

\section{The Solution of Problem 3}

In this section, we discuss the optimal approximation Problem 3. Since the least squares problem is always consistent, it is easy to verify that the solution set $S_{E}$ of Problem 2 is a nonempty convex cone, so the optimal approximation solution is unique.

Without loss of generality, we can assume that the given matrices $\widetilde{Z}_{i} \in \operatorname{CS} R_{\star, q_{i}}^{p_{i} \times p_{i}}$. In fact, from Lemma 4 , arbitrary $\widetilde{Z}_{i} \in$ $R^{p_{i} \times p_{i}}$ can be divided into

$$
\begin{array}{r}
\widetilde{Z}_{i}=\widetilde{Z}_{i 1}+\widetilde{Z}_{i 2}+\widetilde{X}_{i 3}, \quad \text { with } \widetilde{Z}_{i 1} \in \operatorname{CSR}_{\star, q_{i}}^{p_{i} \times p_{i}}, \\
\widetilde{Z}_{i 2} \in \mathrm{CSR}_{\diamond, q_{i}}^{p_{1} \times p_{i}}, \widetilde{Z}_{i 3} \in \mathrm{CASR}^{p_{i} \times p_{i}} .
\end{array}
$$

Furthermore, if $Z_{i} \in \operatorname{CS} R_{\star, q_{i}}^{p_{i} \times p_{i}}$, then

$$
\left\|Z_{i}-\widetilde{Z}_{i}\right\|^{2}=\left\|Z_{i}-\widetilde{Z}_{i 1}\right\|^{2}+\left\|\widetilde{Z}_{i 2}\right\|^{2}+\left\|\widetilde{Z}_{i 3}\right\|^{2},
$$

which meets the claim.

Denote $\bar{Z}_{i}=Z_{i}-\widetilde{Z}_{i}, \bar{F}=F-\mathscr{F}\left(\widetilde{Z}_{1}, \widetilde{Z}_{2}, \ldots, \widetilde{Z}_{t}\right)$, then to solve Problem 3 is equivalent to find the least-norm solution of the new matrix equation

$$
\mathscr{F}\left(\bar{Z}_{1}, \bar{Z}_{2}, \ldots, \bar{Z}_{t}\right)=\bar{F} .
$$

Furthermore, similar to the construction of (11), Problem 2 is transformed equivalently into finding the least-norm leastsquares solution of matrix equation

$$
\mathscr{F}\left(\bar{Y}_{1}, \bar{Y}_{2}, \ldots, \bar{Y}_{t}\right)=\bar{G}, \quad \text { with } \bar{Y}_{i} \in \operatorname{CSR}_{\star, q_{i}}^{p_{i} \times p_{i}}
$$

in which $\bar{G}=\bar{F}-\mathscr{F}\left(Z_{1}^{\diamond}, Z_{2}^{\diamond}, \ldots, Z_{t}^{\diamond}\right)$.

Therefore, we can apply Algorithm 7 to derive the required solution of matrix equation (49). Virtually, it follows from Theorem 13 that if let the initial iteration matrices $\bar{Y}_{i}^{(0)}=$ $\mathscr{L}_{i}\left(M_{i}^{T} \widetilde{H} N_{i}^{T}\right)$ with arbitrary $\widetilde{H} \in R^{m \times n}$, or especially $\bar{Y}_{i}^{(0)}=$ $0 \in R^{p_{i} \times p_{i}}$, then the iteration solutions $\bar{Y}_{i}^{*}$ consist of the leastnorm least-squares solution of which. In this case, the unique optimal approximation solution to Problem 3 can be obtained by

$$
\begin{aligned}
& \left(\widehat{Z}_{1}, \widehat{Z}_{2}, \ldots, \widehat{Z}_{t}\right) \\
& \quad=\left(\bar{Y}_{1}^{*}+\widetilde{Z}_{1}+Z_{1}^{\diamond}, \bar{Y}_{2}^{*}+\widetilde{Z}_{2}+Z_{2}^{\diamond}, \ldots, \bar{Y}_{t}^{*}+\widetilde{Z}_{t}+Z_{t}^{\diamond}\right) .
\end{aligned}
$$

\section{Numerical Example}

In this section, we illustrate the efficiency and reliability of Algorithm 7 by some numerical experiments.

All the numerical experiments are performed by using Matlab 6.5. In addition, because of the influence of the roundoff errors, $P_{i}^{(k)}$ may not equal zero within finite iteration steps, so the iteration will be terminated if $\sum_{i=1}^{t}\left\|P_{i}^{(k)}\right\|^{2}<\epsilon$, for example, let $\epsilon=1.0 e-008$. At this time, $\left(Y_{1}^{(k)}, Y_{1}^{(k)}, \ldots, Y_{t}^{(k)}\right)$ can be regarded as a solution of matrix equation (11), and $Y_{i}^{(k)}+Z_{i}^{\diamond}(i=1,2, \ldots, t)$ consist of the solution group to Problem 2. In particular, let the initial iteration matrices $Y_{i}^{(0)}=0$, then we will obtain the least-norm solution by (36).

Example 1. Input matrices $M_{1}, M_{2}, M_{3}, N_{1}, N_{2}, N_{3}$, and $F$ as follows:

$$
\begin{aligned}
& M_{1}=\left[\begin{array}{cc}
\operatorname{hilb}\left(\frac{r}{2}\right) & \text { ones }\left(\frac{r}{2}\right) \\
\operatorname{hankel}\left(1: \frac{r}{2}\right) & \operatorname{zeros}\left(\frac{r}{2}\right)
\end{array}\right], \\
& M_{2}=\left[\begin{array}{cc}
\text { toeplitz }\left(1: \frac{r}{2}\right) & \text { hilb }\left(\frac{r}{2}\right) \\
\text { ones }\left(\frac{r}{2}\right) & \text { hankel }\left(1: \frac{r}{2}\right)
\end{array}\right] \text {, } \\
& M_{3}=\left[\begin{array}{cc}
\operatorname{zeros}\left(\frac{r}{2}\right) & \text { hankel }\left(1: \frac{r}{2}\right) \\
\operatorname{hilb}\left(\frac{r}{2}\right) & \text { ones }\left(\frac{r}{2}\right)
\end{array}\right] \text {, } \\
& N_{1}=\text { eye }(r), \quad N_{2}=\text { ones }(r) \text {, } \\
& N_{3}=\operatorname{tridiag}([7,1,-1], r) \text {, } \\
& F=\left[\begin{array}{ccccc}
3 & -2 & -1 & & \\
-2 & 3 & -2 & \ddots & \\
-1 & -2 & \ddots & -2 & -1 \\
& \ddots & -2 & 3 & -2 \\
& & -1 & -2 & 3
\end{array}\right]
\end{aligned}
$$

where toeplitz $(k), \operatorname{hilb}(k)$, hankel $(k), \operatorname{zeros}(k)$, and eye $(k)$ 


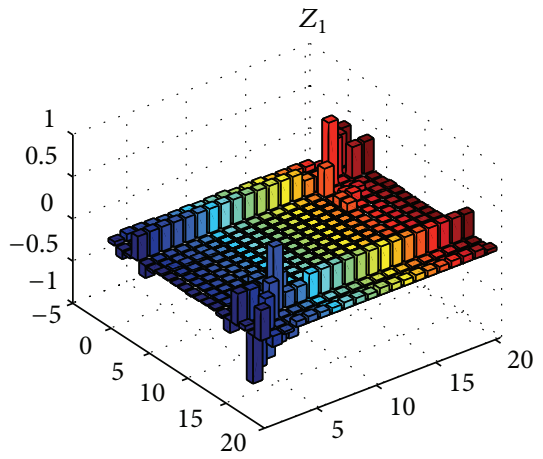

(a)

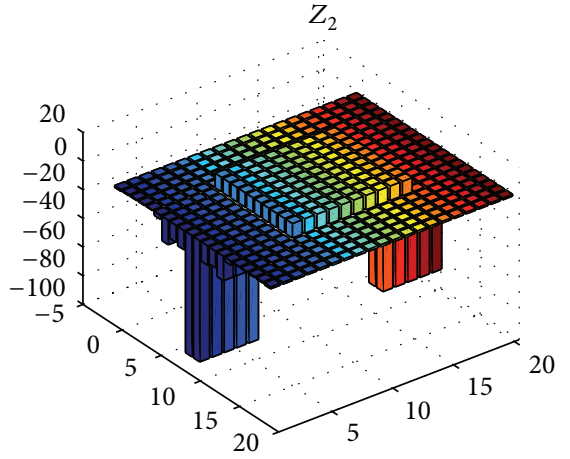

(b)

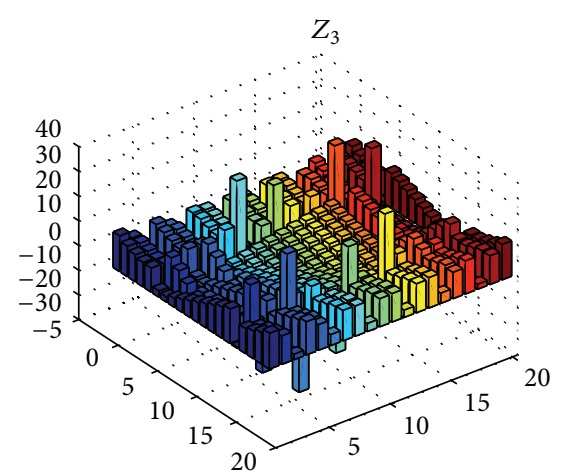

(c)

FIgURE 1: The bars graphs of $Z_{i}$ when $r=20$.

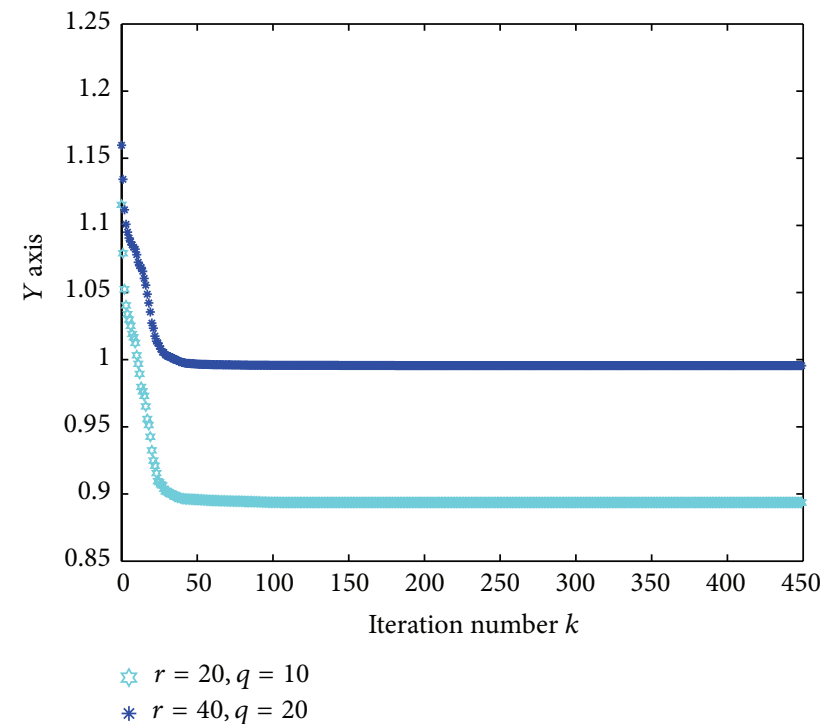

Figure 2: The convergence curve of the Frobenius norm of the residual.

denote the Toeplitz matrix, Hilbert matrix, Hankel matrix, null matrix, identity matrix with orders $k$, and the elements of matrix ones $(\cdot)$ are one, tridiag $([7,1,-1], r)$ represents $r \times r$ tri-diagonal matrix produced by vector $[7,1,-1]$.

Let the given central principal matrices

$$
\begin{gathered}
\mathscr{X}_{1}=\operatorname{zeros}\left(\frac{r}{2}\right), \\
\mathscr{X}_{2}=\text { ones }\left(\frac{r}{2}\right) * 10, \quad \mathscr{X}_{3}=\text { toeplitz }\left(1: \frac{r}{2}\right) .
\end{gathered}
$$

By using the Algorithm 7, we obtain the solution to Problem 2. To save space, we shall not report the explicit datum of the solution, but the bars graphs of the components for the solution matrices will be given. Let $r=20$, Figure 1 shows the

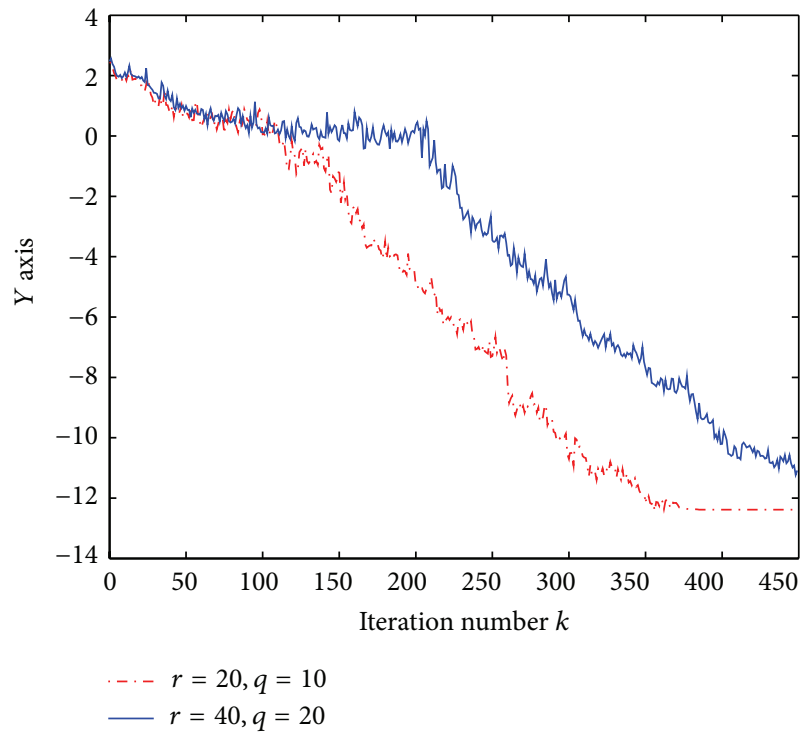

FIgURE 3: The convergence curve of the Frobenius norm of the terminated condition.

bars graphs of $Z_{1}, Z_{2}, Z_{3}$ when we choose the initial iterative matrices

$$
Y_{i}=\left(\begin{array}{ccc}
\text { ones }\left(\frac{r-q}{2}\right) & \text { ones }\left(\frac{r-q}{2}, q\right) & \text { ones }\left(\frac{r-q}{2}\right) \\
\text { ones }\left(q, \frac{r-q}{2}\right) & \operatorname{zeros}\left(\frac{r}{2}\right) & \text { ones }\left(q, \frac{r-q}{2}\right) \\
\text { ones }\left(\frac{r-q}{2}\right) & \text { ones }\left(\frac{r-q}{2}, q\right) & \text { ones }\left(\frac{r-q}{2}\right)
\end{array}\right) \text {, }
$$

$i=1,2,3$,

and the terminal condition $\left\|P_{1}^{(k)}\right\|+\left\|P_{2}^{(k)}\right\|+\left\|P_{3}^{(k)}\right\|<\epsilon=$ $1.0 e-012$.

Moreover, when $r=20$ and $r=40$, the convergence curves for the Frobenius norm of the residual denoted by RES $=\left\|R_{k}\right\|$ and the termination condition denoted by $\mathrm{TC}=\left\|P_{1}^{(k)}\right\|+\left\|P_{2}^{(k)}\right\|+\left\|P_{3}^{(k)}\right\|$ are plotted in Figures 2 and 3 , respectively. 
From Figure 2, we can see that the residual norm of Algorithm 7 is monotonically decreasing, which is in accordance with the theory established in Theorem 14, namely, this algorithm is numerical stable. While Figure 3 shows that the terminated condition $\left\|P_{1}^{(k)}\right\|+\left\|P_{2}^{(k)}\right\|+\left\|P_{3}^{(k)}\right\|$ is oscillating back and forth and approaches to zero as iterative process. Hence, the iterative Algorithm 7 is efficient, but it lacks of smooth convergence. Of course, for a problem with large and sparse matrices, Algorithm 7 may not terminate in a finite number of steps because of roundoff errors. How to establish an efficient and smooth algorithm is an important problem which we should study in a future work.

\section{Conflict of Interests}

The authors declare that there is no conflict of interests regarding the publication of this paper.

\section{Acknowledgments}

The authors would like to express their sincere gratitude to the editor and two anonymous reviewers for their valuable comments and suggestions which have helped immensely improving the quality of the paper. Mao-lin Liang acknowledges the support of the scientific foundation of Tianshui Normal University (no. TSA1104). Young-hong Shen is supported by the "QingLan" Talent Engineering Funds of Tianshui Normal University.

\section{References}

[1] G. H. Golub and C. F. Van Loan, Matrix Computations, John Hopkins University Press, Baltimore, Md, USA, 1996.

[2] L. Datta and S. D. Morgera, "On the reducibility of centrosymmetric matrices-applications in engineering problems," Circuits, Systems, and Signal Processing, vol. 8, no. 1, pp. 71-96, 1989.

[3] J. Respondek, "Controllability of dynamical systems with constraints," Systems \& Control Letters, vol. 54, no. 4, pp. 293-314, 2005.

[4] I. S. Pressman, "Matrices with multiple symmetry properties: applications of centro-Hermitian and per-Hermitian matrices," Linear Algebra and its Applications, vol. 284, no. 1-3, pp. 239$258,1998$.

[5] F.-Z. Zhou, X.-Y. Hu, and L. Zhang, "The solvability conditions for the inverse eigenvalue problems of centro-symmetric matrices," Linear Algebra and Its Applications, vol. 364, pp. 147-160, 2003.

[6] D. Boley and G. H. Golub, "A survey of matrix inverse eigenvalue problems," Inverse Problems, vol. 3, no. 4, pp. 595-622, 1987.

[7] Z.-J. Bai, "The inverse eigenproblem of centrosymmetric matrices with a submatrix constraint and its approximation," SIAM Journal on Matrix Analysis and Applications, vol. 26, no. 4, pp. 1100-1114, 2005.

[8] C. C. Paige, "Computing the generalized singular value decomposition," Society for Industrial and Applied Mathematics, vol. 7, no. 4, pp. 1126-1146, 1986.

[9] X.-P. Pan, X.-Y. Hu, and L. Zhang, "A class of constrained inverse eigenproblem and associated approximation problem for skew symmetric and centrosymmetric matrices," Linear Algebra and its Applications, vol. 408, pp. 66-77, 2005.

[10] F.-Z. Zhou, L. Zhang, and X.-Y. Hu, "Least-square solutions for inverse problems of centrosymmetric matrices," Computers \& Mathematics with Applications, vol. 45, no. 10-11, pp. 1581-1589, 2003.

[11] F.-L. Li, X.-Y. Hu, and L. Zhang, "Left and right inverse eigenpairs problem of skew-centrosymmetric matrices," Applied Mathematics and Computation, vol. 177, no. 1, pp. 105-110, 2006.

[12] O. Rojo and H. Rojo, "Some results on symmetric circulant matrices and on symmetric centrosymmetric matrices," Linear Algebra and its Applications, vol. 392, pp. 211-233, 2004.

[13] D. X. Xie, X. Y. Hu, and Y.-P. Sheng, "The solvability conditions for the inverse eigenproblems of symmetric and generalized centro-symmetric matrices and their approximations," Linear Algebra and Its Applications, vol. 418, no. 1, pp. 142-152, 2006.

[14] Z. Y. Liu and H. Faßbender, "Some properties of generalized $K$-centrosymmetric $H$-matrices," Journal of Computational and Applied Mathematics, vol. 215, no. 1, pp. 38-48, 2008.

[15] F.-L. Li, X.-Y. Hu, and L. Zhang, "Left and right inverse eigenpairs problem of generalized centrosymmetric matrices and its optimal approximation problem," Applied Mathematics and Computation, vol. 212, no. 2, pp. 481-487, 2009.

[16] M.-L. Liang, C.-H. You, and L.-F. Dai, “An efficient algorithm for the generalized centro-symmetric solution of matrix equation $A X B=C$, Numerical Algorithms, vol. 44, no. 2, pp. 173-184, 2007.

[17] Q. X. Yin, "Construction of real antisymmetric and biantisymmetric matrices with prescribed spectrum data," Linear Algebra and Its Applications, vol. 389, pp. 95-106, 2004.

[18] P. Deift and T. Nanda, "On the determination of a tridiagonal matrix from its spectrum and a submatrix," Linear Algebra and its Applications, vol. 60, pp. 43-55, 1984.

[19] L. S. Gong, X. Y. Hu, and L. Zhang, "The expansion problem of anti-symmetric matrix under a linear constraint and the optimal approximation," Journal of Computational and Applied Mathematics, vol. 197, no. 1, pp. 44-52, 2006.

[20] Y. X. Yuan and H. Dai, "Inverse problems for symmetric matrices with a submatrix constraint," Applied Numerical Mathematics, vol. 57, no. 5-7, pp. 646-656, 2007.

[21] L. J. Zhao, X. Y. Hu, and L. Zhang, "Least squares solutions to $A X=B$ for bisymmetric matrices under a central principal submatrix constraint and the optimal approximation," Linear Algebra and its Applications, vol. 428, no. 4, pp. 871-880, 2008.

[22] A.-P. Liao and Y. Lei, "Least-squares solutions of matrix inverse problem for bi-symmetric matrices with a submatrix constraint," Numerical Linear Algebra with Applications, vol. 14, no. 5, pp. 425-444, 2007.

[23] G. P. Xu, M. S. Wei, and D. S. Zheng, "On solutions of matrix equation $A X B+C Y D=F$, Linear Algebra and Its Applications, vol. 279, no. 1-3, pp. 93-109, 1998.

[24] B. Zhou and G.-R. Duan, "On the generalized Sylvester mapping and matrix equations," Systems \& Control Letters, vol. 57, no. 3, pp. 200-208, 2008.

[25] A.-G. Wu, G. Feng, G.-R. Duan, and W.-J. Wu, "Finite iterative solutions to a class of complex matrix equations with conjugate and transpose of the unknowns," Mathematical and Computer Modelling, vol. 52, no. 9-10, pp. 1463-1478, 2010.

[26] F. Ding and T. Chen, "Iterative least-squares solutions of coupled Sylvester matrix equations," Systems \& Control Letters, vol. 54, no. 2, pp. 95-107, 2005. 
[27] Z.-H. Peng, X.-Y. Hu, and L. Zhang, "The bisymmetric solutions of the matrix equation $A_{1} X_{1} B_{1}+A_{2} X_{2} B_{2}+\cdots+A_{l} X_{l} B_{l}=C$," Linear Algebra and its Applications, vol. 426, no. 2-3, pp. 583595, 2007.

[28] M. Dehghan and M. Hajarian, "The general coupled matrix equations over generalized bisymmetric matrices," Linear Algebra and Its Applications, vol. 432, no. 6, pp. 1531-1552, 2010.

[29] J.-F. Li, X.-Y. Hu, and L. Zhang, "The submatrix constraint problem of matrix equation $A X B+C Y D=E$," Applied Mathematics and Computation, vol. 215, no. 7, pp. 2578-2590, 2009.

[30] T. Meng, "Experimental design and decision support," in Expert Systems, the Technology of Knowledge Management and Decision Making-Forthe 21st Century, C. Leondes, Ed., Academic Press, 2001.

[31] M. Baruch, "Optimization procedure to correct stiffness and flexibility matrices using vibration tests," AIAA Journal, vol. 16, no. 11, pp. 1208-1210, 1978.

[32] N. J. Higham, "Computing a nearest symmetric positive semidefinite matrix," Linear Algebra and its Applications, vol. 103, pp. 103-118, 1988.

[33] Z.-Y. Peng, X.-Y. Hu, and L. Zhang, "The nearest bisymmetric solutions of linear matrix equations," Journal of Computational Mathematics, vol. 22, no. 6, pp. 873-880, 2004.

[34] A.-P. Liao, Z.-Z. Bai, and Y. Lei, "Best approximate solution of matrix equation $A X B+C Y D=E$," SIAM Journal on Matrix Analysis and Applications, vol. 27, no. 3, pp. 675-688, 2005.

[35] Z.-Y. Peng and Y.-X. Peng, "An efficient iterative method for solving the matrix equation $A X B+C Y D=E$," Numerical Linear Algebra with Applications, vol. 13, no. 6, pp. 473-485, 2006.

[36] Y.-B. Deng, Z.-Z. Bai, and Y.-H. Gao, "Iterative orthogonal direction methods for Hermitian minimum norm solutions of two consistent matrix equations," Numerical Linear Algebra with Applications, vol. 13, no. 10, pp. 801-823, 2006. 


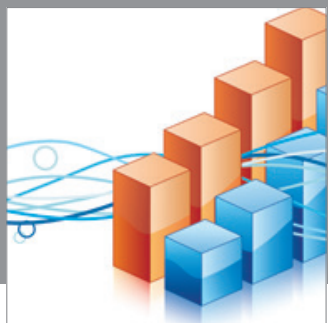

Advances in

Operations Research

mansans

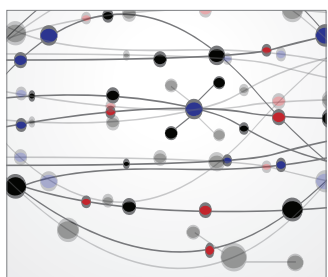

The Scientific World Journal
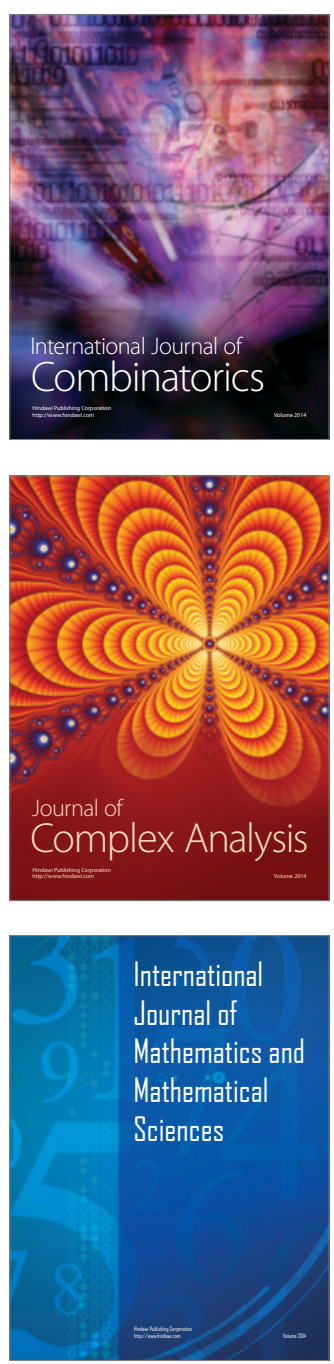
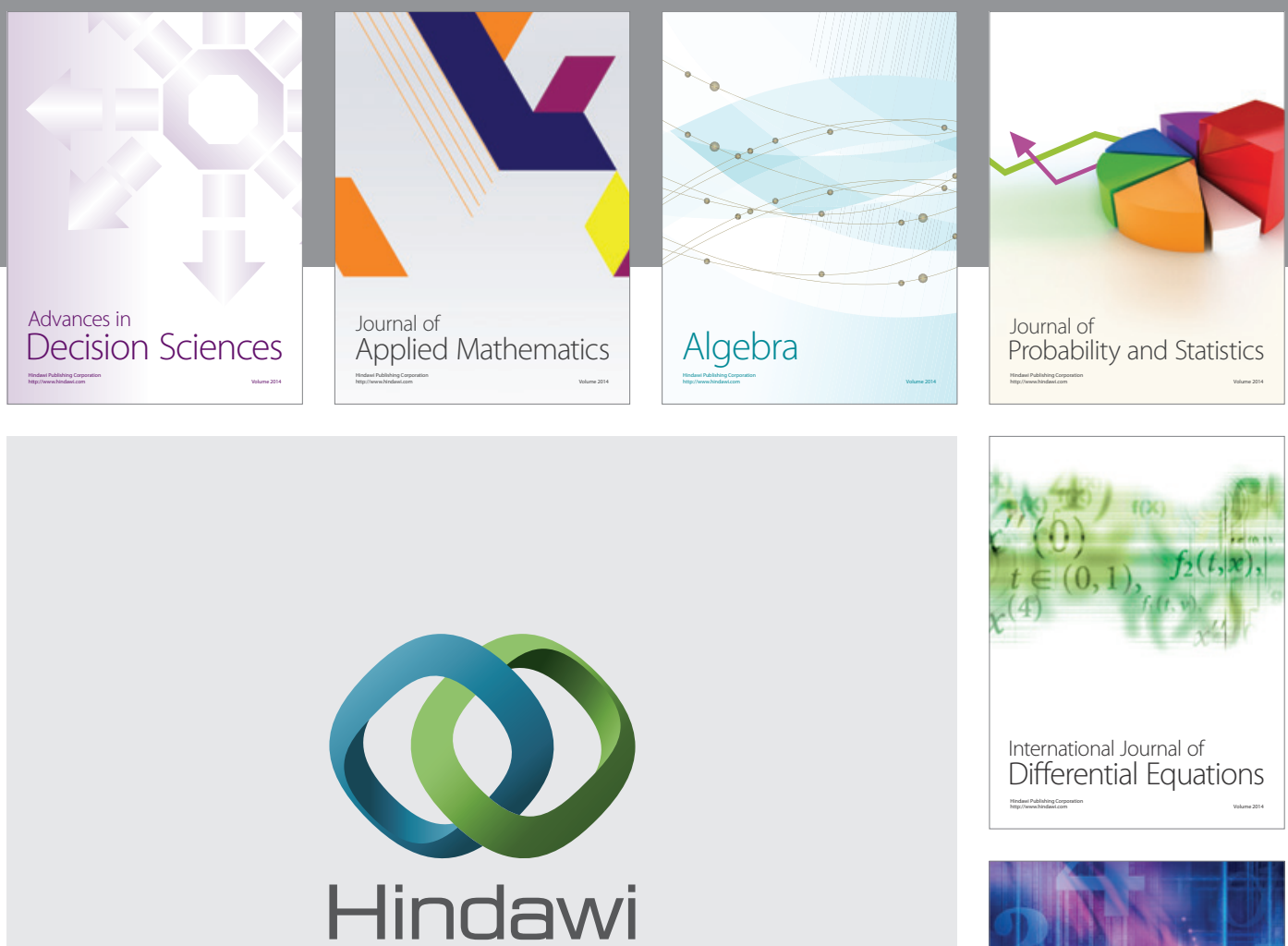

Submit your manuscripts at http://www.hindawi.com
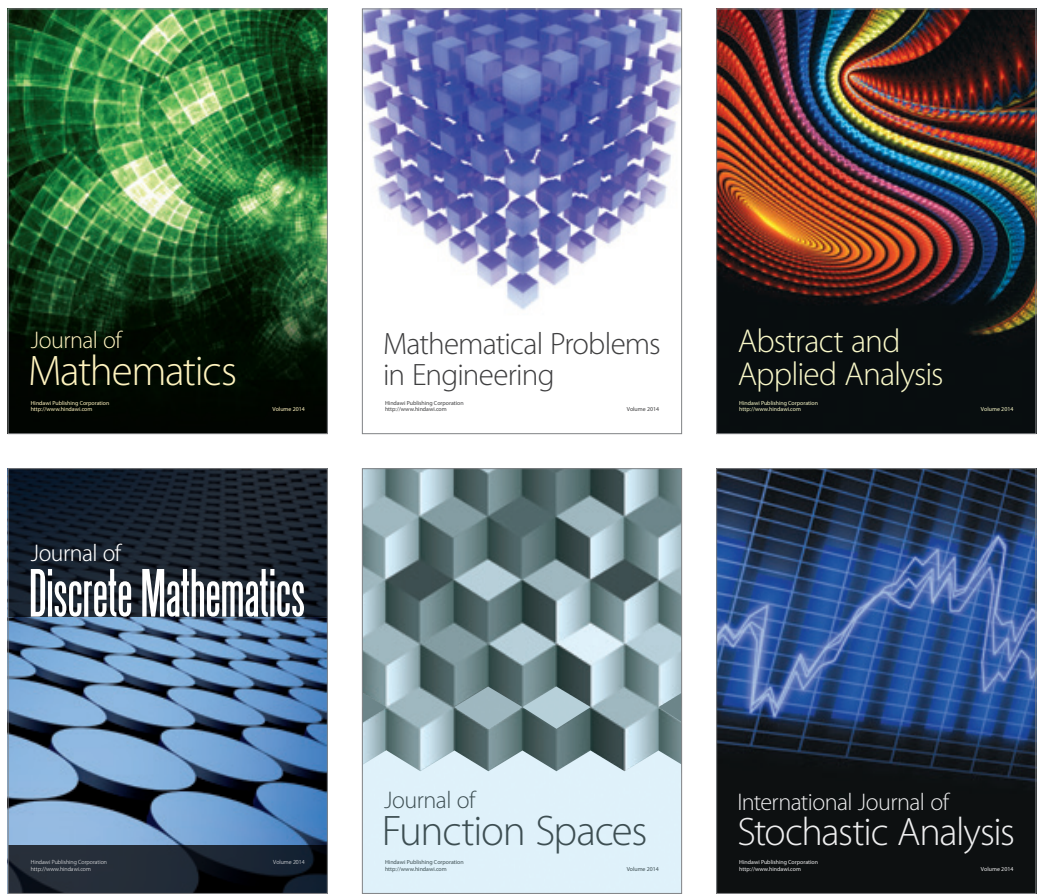

Journal of

Function Spaces

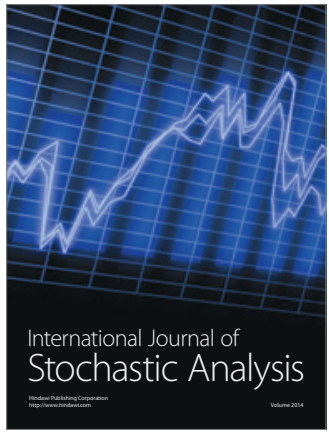

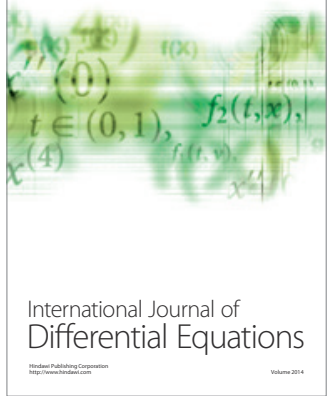
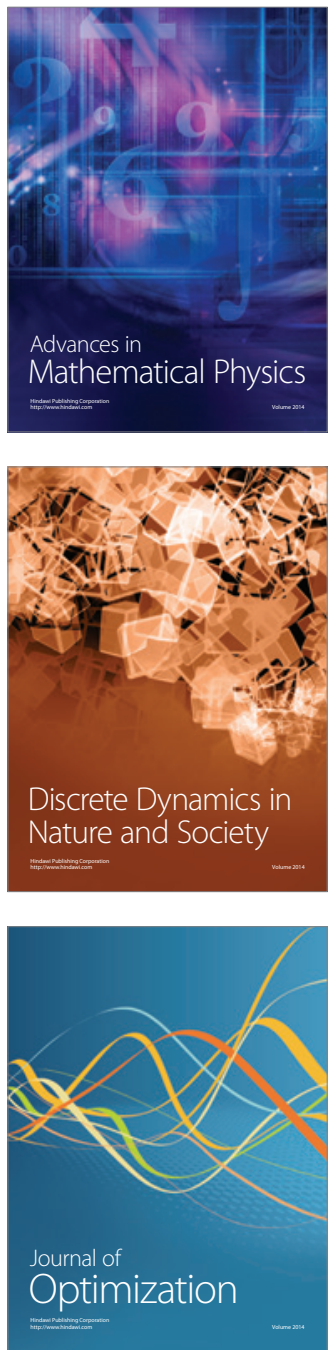